\title{
An innovative rotational Raman lidar to measure the temperature profile from the surface to $30 \mathrm{~km}$ altitude
}

\author{
Alain Hauchecorne ${ }^{1 *}$, Philippe Keckhut ${ }^{1}$, Jean-François Mariscal ${ }^{1}$, Eric d'Almeida ${ }^{1}$, Pierre- \\ Richard Dahoo ${ }^{1}$, Jacques Porteneuve ${ }^{1}$ \\ ${ }^{1}$ LATMOS-IPSL, Université Versailles St-Quentin, CNRS, Guyancourt, France, *Email: \\ alain.hauchecorne@latmos.ipsl.fr
}

\begin{abstract}
A concept of innovative rotational Raman lidar with daylight measurement capability is proposed to measure the vertical profile of temperature from the ground to the middle stratosphere. The optical filtering is made using a Fabry-Pérot Interferometer with line spacing equal to the line spacing of the Raman spectrum. The detection is made using a linear PMT array operated in photon counting mode. We plan to build a prototype and to test it at the Haute-Provence Observatory lidar facility. to achieve a time resolution permitting the observation of small-scale atmospheric processes playing a role in the troposphere-stratosphere interaction as gravity waves. If successful, this project will open the possibility to consider a Raman space lidar for the global observation of atmospheric temperature profiles.
\end{abstract}

\section{INTRODUCTION}

The temperature is a fundamental parameter for meteorology and climatology. It is measured, (1) twice a day using radiosondes with good vertical resolution but in a limited number of stations in the world and, (2) from space but often with a vertical resolution limited by the weighting function of the instrument. Lidars provide a good vertical resolution and continuity of measurements. Currently the temperature is measured by Rayleigh lidar at NDACC ground stations but only above $30 \mathrm{~km}$ due to the presence of aerosols below this altitude [1] [2]. There is currently no space lidar in flight or in project measuring the temperature. The rotational Raman lidar technology allows access to the evolution of the temperature profile in the troposphere and lower stratosphere. It requires developing an efficient optical system with separation between the Rayleigh and the rotational Raman signal, the first being 2 to 3 orders of magnitude stronger than the second. It requires acquiring the full rotational Raman spectrum of molecular nitrogen, the temperature being deduced from the evolution of the relationship between the spectral lines.

\section{METHODOLOGY}

In the Rayleigh lidar, monochromatic laser pulses are sent vertically into the atmosphere and a temporal analysis of the backscattered light provides information about the vertical structure and composition of the atmosphere. Above the top of the stratospheric aerosol layer (about $30 \mathrm{~km}$ ), Mie scattering is negligible and the received signal due only to Rayleigh scattering is directly proportional to the atmospheric density. The temperature profile is deduced from the density profile assuming that the density is in hydrostatic equilibrium and obeys the perfect gas law. The profile is limited upwards by the signal to noise ratio at about $80-90 \mathrm{~km}$, depending on the power of the lidar.

It is obvious that below about $30 \mathrm{~km}$, depending on the height and abundance of aerosols, it is not possible to use the Rayleigh scattering technique to measure atmospheric density and temperature because of the contribution of Mie scattering to the signal. It is, however, possible to use the Raman vibrational and rotational components of the scattering by the main gases of the atmosphere, $\mathrm{O}_{2}$ and $\mathrm{N}_{2}$. The vibrational Raman scattering is about 3 orders of magnitude smaller than the Rayleigh scattering. In order to achieve a useful accuracy in the lower stratosphere with this technique, it is then necessary to have a lidar with high energy-surface product.

As for the Rayleigh scattering, the vibrational Raman signal is directly proportional to the atmospheric density and the temperature profile is computed from the density profile assuming the hydrostatic equilibrium. It is necessary to correct the signal for the atmospheric transmission, which cannot be assumed constant below $30 \mathrm{~km}$. This 
technique is very well adapted for observations in the lower stratosphere during periods with low background aerosol loading during which an estimation of the optical thickness of the aerosol layer can be done with enough accuracy [3].

The vibrational Raman technique is limited to the altitude above the tropopause due to the frequent presence of thin cirrus with unknown optical thickness in the upper troposphere. Furthermore, after a major volcanic eruption, like the Pinatubo in June 1991, it is no more possible to use the vibrational Raman technique in the lower stratosphere due to the uncertainty on the very high optical thickness of the volcanic aerosol layer. In this case an alternative technique exists consisting in the direct measurement of the temperature using the rotational Raman lidar technique.

The Raman rotational spectrum of a diatomic molecule such as $\mathrm{N}_{2}$ or $\mathrm{O}_{2}$ is composed of two branches, Stokes and anti-Stokes. Each branch is composed of a series of lines with an intensity depending on temperature. Lines close to the Rayleigh line vary inversely to the temperature when liens far to the Rayleigh line vary in phase with the temperature. If we measure the ratio between two lines with an opposite dependence in temperature, we can obtain a direct measurement of the atmospheric temperature. This is generally done with two anti-Stokes lines near $529 \mathrm{~nm}$ and $530.5 \mathrm{~nm}$ [4] [5] [6]. Anti-Stokes lines are preferred to the Stokes lines that may be perturbed by the fluorescence of aerosols. The difficulty of these measurements is due to the very small shift of wavelength between the laser excitation line $(532 \mathrm{~nm})$ and the observed lines. The needed rejection factor at the wavelength of the laser line is of the order of $10^{-7}$ and is obtained by using two interference filters in series. This method gives a relative temperature profile and has to be calibrated once with a radiosonde profile to obtain the two parameters of the calibration curve. This curve can be used as long as the characteristics of the lidar are not changed.

Figure 1 presents a profile obtained at HauteProvence Observatory (OHP, $\left.44^{\circ} \mathrm{N}, 6^{\circ} \mathrm{E}\right)$ integrated during $10 \mathrm{~h}$ with a $1-\mathrm{km}$ vertical resolution, showing a good agreement with the coincident radiosonde. This technique has been installed in the ALOMAR lidar facility (Andoya Space Center, $69^{\circ} \mathrm{N}, 16^{\circ} \mathrm{E}$ ) in 1995 and is operated regularly since this date. It has been used to study the properties of polar stratospheric clouds, showing the capability to obtain temperature profiles inside the clouds [6]. However the low level of the signal after filtering by the two band-pass filters limits the use of the rotational Raman technique for dynamics studies in the lower stratosphere that need e better time resolution. For instance for the study of gravity waves a time resolution less than 30 minutes is needed.

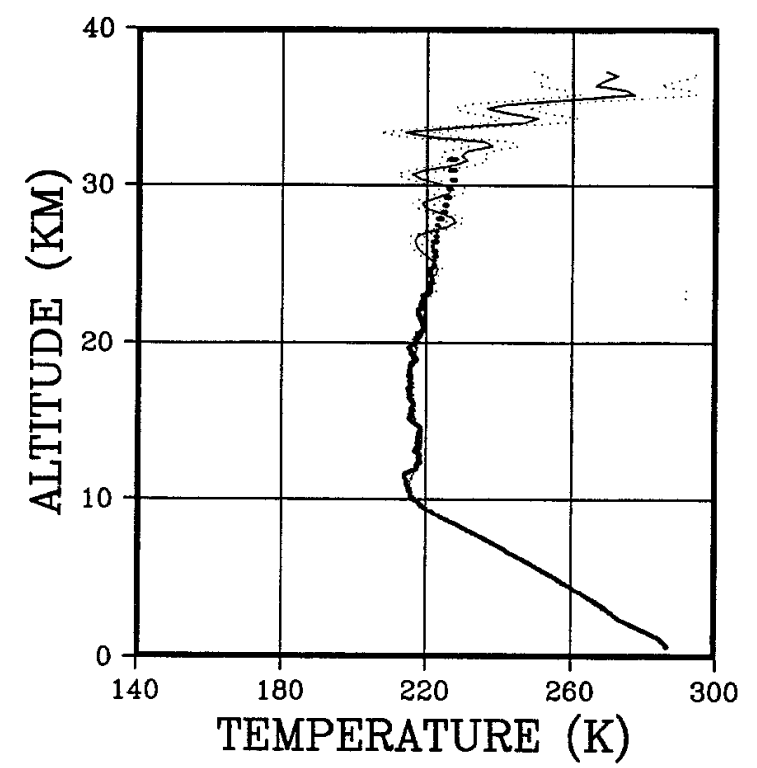

Figure 1: Rotational Raman profile at OHP on April 9, 1991: lidar (thin line) with $1 \sigma$ uncertainty (dotted line) compared with a radiosonde (thick line). From [4].

We propose to develop an innovative rotational Raman lidar using the signal recorded in the full rotational Raman spectrum. The integrated intensity of the full $\mathrm{N}_{2}$ rotational Raman spectrum represents $2 \%$ of the intensity of the Rayleigh line, which is not at all negligible. In order to do that, it is needed to acquire the very low backscatter signal at the frequency required to achieve the expected vertical resolution $(1 \mu$ s for $150 \mathrm{~m}$ ). New array detectors, like the Hamamatsu 32-channnel linear array PMT assembly H7260 operated in photon counting mode, are well adapted for this objective. 
Another challenge is the high dynamics between the Rayleigh-Mie line and rotational Raman lines that are individually 3 to 4 orders of magnitude weaker. An elegant solution to solve this problem proposed by [7] is to transmit the optical signal through a Fabry-Pérot interferometer (FPI) with a line spectral spacing exactly equal to the spacing between the lines of the $\mathrm{N}_{2}$ rotational Raman spectrum $(7.958 \mathrm{~cm}-1$ [6]). The Rayleigh line is placed exactly in the middle of two FPI lines, corresponding to a minimum of transmission. For a FPI with finesse 30, the intensity of the Rayleigh-Mie line is attenuated by a factor 160 and is only 6 times larger than the stronger Raman lines. The use of a FPI has another advantage. It decreases the sky background light by a factor approximately equal to its finesse, giving to the Raman lidar a daylight measurement capability.

We started the definition of the rotational Raman detection system. The objective is to build a prototype and to test it in the NDACC station at Haute-Provence Observatory to benefit from the facilities available on site (laser source, telescopes, ...). Such a system will be able to perform a continuous survey of the temperature profile from the surface to the middle stratosphere, providing a powerful tool to study the variability of the temperature profiles due to atmospheric processes and could serve as a reference to define a future space lidar for measuring the temperature in the troposphere and lower stratosphere.

\section{CONCLUSION}

We propose an innovative rotational Raman lidar with daylight measurement capability to measure continuously the vertical profile of temperature from the ground to the middle stratosphere. The optical filtering is made using a FPI with line spacing equal to the line spacing of the $\mathrm{N}_{2}$ rotational Raman spectrum. This allows benefiting from the signal in the whole $\mathrm{N}_{2}$ rotational Raman spectrum and achieving a time resolution permitting the observation of small-scale atmospheric processes playing a role in the troposphere-stratosphere interaction as upward propagating gravity waves. If successful, this project will open the possibility to consider a rotational Raman space lidar for the global observation of atmospheric temperature profiles with a high vertical resolution.

\section{ACKNOWLEDGEMENT}

This work is made with the support from CNRSINSU, CNES and UVSQ.

\section{REFERENCES}

[1] Hauchecorne A., M.L. Chanin, Density and temperature profiles obtained by lidar between 35 and 70 km, Geophys. Res. Lett., 7, 565-568, 1980.

[2] Keckhut P., A. Hauchecorne, M.L. Chanin, A critical review of the data base acquired for the long term surveillance of the middle atmosphere by Rayleigh lidar, J. Atm. Ocean. Tech., 10, 850-867, 1993.

[3] Keckhut P., M.L. Chanin, A. Hauchecorne, Stratospheric temperature measurements using Raman lidar, Applied Optics, 29, 5182-5186, 1990.

[4] Hauchecorne A., M.L. Chanin, P.Keckhut, and D. Nedeljkovic, Lidar monitoring of the temperature in the middle atmosphere, Appl. Phys. B, 55, 29-34, 1992.

[5] Nedeljkovic N., A. Hauchecorne, M.L. Chanin, Rotational Raman lidar to measure the atmospheric temperature from the ground to $30 \mathrm{~km}$, IEEE Transactions on Geoscience and Remote Sensing, 31, 91-101, 1993.

[6] Behrendt, A., J. Reichardt, Atmospheric temperature profiling in the presence of clouds with a pure rotational Raman lidar by use of an interferencefilter-based polychromator, Applied Optics, 39 (9), 1372-1378, 2000.

[7] Fierli, F., A. Hauchecorne and B. Knudsen, Analysis of polar stratospheric clouds formation using temperature and aerosols measured by ALOMAR RMR lidar, J. Geophys. Res., 106, 24127-24141.2001.

[8] Arshinov, Y., S/ Bobrovnikov, I. Serikov, A. Ansmann, U. Wandinger, D. Althausen, I. Mattis, D. Müller Daytime operation of a pure rotational Raman lidar by use of a Fabry-Perot interferometer, Applied Optics, 2005, 44, 3593-3603, 2005. 